\section{Label-free and highly sensitive optical imaging of detailed microcirculation within meninges and cortex in mice with the cranium left intact}

\author{
Yali Jia, Lin An, and Ruikang K. Wang \\ Oregon Health \& Science University, Department of \\ Biomedical Engineering, 3303 SW Bond Avenue, Portland, \\ Oregon 97239
}

\begin{abstract}
We demonstrate for the first time that the detailed blood flow distribution within intracranial dura mater and cortex can be visualized by an ultrahigh sensitive optical microangiography (UHS-OMAG). The study uses an UHS-OMAG system operating at $1310 \mathrm{~nm}$ with an imaging speed at 150 frames per second that requires $\sim 10$ s to complete one $3-D$ scan of $\sim 2.5 \times 2.5 \mathrm{~mm}^{2}$. The system is sensitive to blood flow with a velocity ranging from $\sim 4 \mu \mathrm{m} / \mathrm{s}$ to $\sim 23 \mathrm{~mm} / \mathrm{s}$. We show superior performance of UHS-OMAG in providing functional images of capillary level microcirculation within meninges in mice with the cranium left intact, the results of which correlate well with the standard dural histopathology. ๑ 2010 Society of Photo-Optical Instrumentation Engineers. [DOI: 10.1117/1.3432654]
\end{abstract}

Keywords: optical microangiography; microcirculation; blood flow; meningeal blood vessel network.

Paper 10164LR received Mar. 26, 2010; revised manuscript received Apr. 22, 2010; accepted for publication Apr. 26, 2010; published online May 25, 2010

The highly vascularized central nervous system (CNS) is enclosed by three connective tissue layers: dura mater (innermost layer), arachnoid mater (middle layer), and pia mater (outmost layer), which collectively are called meninges. Being a fibrous membrane, the dura mater has unexpectedly rich blood supply, despite the fact that the meningeal arteries in the dura mainly supply the cranium. ${ }^{1}$ The vascularization and blood circulation within the intracranial dura mater can play an important role in a number of neurological diseases and complications. ${ }^{2}$ For example, the meningeal vasodilation/ constriction is thought to be responsible for migraine, ${ }^{3}$ the hypervascularization within meninges may attribute to meningioma; ${ }^{2}$ and the rupture of meningeal blood vessels could lead to epidural or subdural hemorrhage. ${ }^{4}$ To improve our fundamental understanding of mechanisms involved with these neurological complications, there is a need for an imaging tool that can functionally image the detailed microcirculation within the meninges alone, without contamination from the microcirculation below, i.e., from cortical vessels. In addition, such an imaging tool would also shed light on how the

Address all correspondence to: E-mail: r.k.wang@bme.ogi.edu microcirculations within the cortex and the meninges would separately contribute to dura-related neurovascular disorders.

Optical microangiography $(\mathrm{OMAG})^{5}$ is a novel extension of optical coherence tomography technology, ${ }^{6,7}$ capable of producing 3-D images of dynamic blood perfusion within microcirculatory tissue beds at an imaging depth up to approximately one transport mean free path below the surface $(\sim 2 \mathrm{~mm}$ in brain). It produces imaging contrasts via endogenous light scattering from flowing blood cells within open vessels; thus, it is a label-free imaging technology. Previously, we have demonstrated the feasibility of OMAG in imaging the cerebral microcirculation in mice with the cranium left intact. ${ }^{8}$ Due to its depth-resolved imaging capability, studies have also shown that OMAG was able to separate the blood circulations within meninges from those in the cortex. ${ }^{9,10}$ In these studies, the rich capillary loops within meninges were not imaged, even though large vessels such as meningeal arterioles and venules were clearly imaged. This failure is attributed to the reported OMAG system only being capable of imaging minimal blood flow at $\sim 160 \mu \mathrm{m} / \mathrm{s}$, limited by the optical heterogenous property of the cranium and brain tissue. ${ }^{10,11}$ However, the blood flow in meningeal vessels, particularly in capillaries, is slow, ranging from tens of microns per second (in capillaries) to millimeters per second (in large vessels). ${ }^{12}$ To visualize the capillary plexus in dura matter, we designed an improved and ultrahigh sensitive OMAG (UHSOMAG) system.

The system setup used to achieve UHS-OMAG is shown in Fig. 1(a). Briefly, a broadband infrared superluminescent diode (SLD) with a central wavelength of $1310 \mathrm{~nm}$ and a spectral bandwidth of $56 \mathrm{~nm}$ was used as the illuminating light source. The light from the SLD was coupled into a fiberbased Michelson interferometer. The light emerging at the output of the interferometer was sent to a custom-built highspeed spectrometer. The spectral resolution of the designed spectrometer was around $0.141 \mathrm{~nm}$, which provided a total depth range of $\sim 3.0 \mathrm{~mm}$ in air $(2.22 \mathrm{~mm}$ in biological tissue by assuming that the refractive index of the sample is $\sim 1.35$ ). The axial resolution of the system was $13 \mu \mathrm{m}$ in air ( $\sim 9.6 \mu \mathrm{m}$ in tissue). To match the lateral resolution with this axial resolution while keeping sufficient depth of focus for imaging meningeal vessels through the intact cranium, we improved the sampling arm optics by having a beam diameter of $\sim 3 \mathrm{~mm}$ and using an objective lens with a focal length of $30 \mathrm{~mm}$ [Fig. 1(b)]. With this modification, the system provided a measured lateral resolution of $\sim 9 \mu \mathrm{m}$ (close to the theoretical value of $8 \mu \mathrm{m}$ according to Abbe's rule) and a depth of focus of $\sim 400 \mu \mathrm{m}$. Because the average size of a capillary vessel is about $10 \mu \mathrm{m}$, we expect that the designed spatial resolution $(\sim 10 \mu \mathrm{m}$ in $x-y-z)$ would be able to image the capillaries in the intracranial dura matter. The imaging speed of the system was 47,000 A scans per second, with which the measured signal-to-noise ratio (SNR) was $\sim 105 \mathrm{~dB}$ at a focus spot of the sampling beam, which was $\sim 500 \mu \mathrm{m}$ below the zero delay line.

Unlike conventional OMAG ${ }^{5}$ to achieve ultrahigh sensitive imaging of slow microcirculation, UHS-OMAG employed a new scanning protocol. First, we acquired a low density B-scan frame (i.e., $x$-direction scan), which encom-

1083-3668/2010/15(3)/030510/3/\$25.00 @ 2010 SPIE 


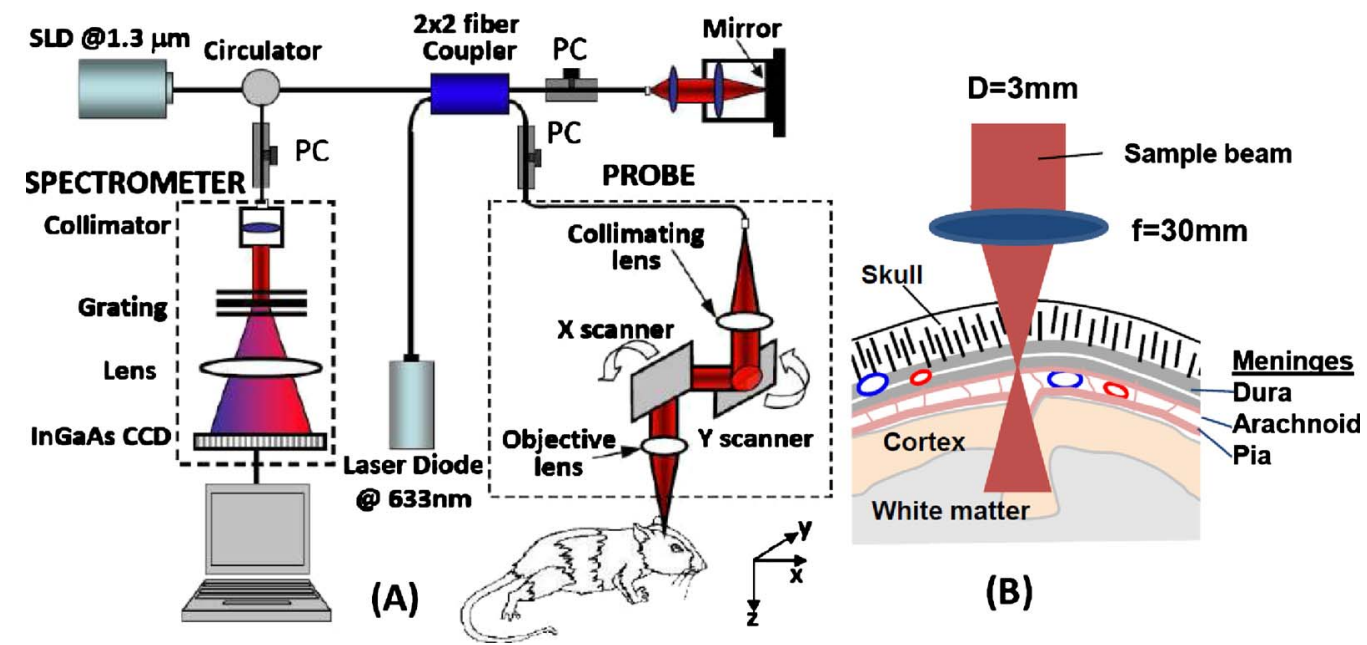

Fig. 1 (a) Schematic of the UHS-OMAG system where PC represents the polarization controller. (b) Sketch of the designed probe beam targeting the meninges (drawing not to scale).

passed 256 A scans with a spacing of $\sim 10 \mu \mathrm{m}$ between adjacent scans that covered a range of $\sim 2.56 \mathrm{~mm}$. With this setup, the system imaging frame rate was set at 150 frames per second (fps). Secondly, to minimize the noise originated from the tissue heterogeneity, we captured a high density $\mathrm{C}$ scan (i.e., $y$-direction scan) consisting of $1500 \mathrm{~B}$ scans over $2.56 \mathrm{~mm}$. Accordingly, the whole 3-D data volume was captured in $10 \mathrm{~s}$. To image the slow flows, UHS-OMAG applied high-pass filtering along the $\mathrm{C}$-scan direction, rather than the B-scan direction as in conventional OMAG. ${ }^{5}$ By using highpass filtering to isolate optical scattering signals between the static and moving scatters, the detectable blood flow of the UHS-OMAG system would range from $\sim 4 \mu \mathrm{m} / \mathrm{s}$ to $\sim 23 \mathrm{~mm} / \mathrm{s}$, a range sufficient to image the blood supply in the meninges.

To test the performance of the UHS-OMAG imaging of dural as well as cortical vascular networks in vivo, threemonth-old C57 BL/6 mice (20 to $30 \mathrm{~g}$ ) were used in the study. The animal imaging protocol has been fully described in Ref. 8. After the scalp was pulled aside, the animal was positioned under the scanning probe, with the meninges carefully placed within the depth of focus of the probe beam [see Fig. 1(b)] as monitored by real-time OMAG/OCT structural images displayed on the computer screen on the fly. The depth-resolved imaging results for blood perfusion within an area of $\sim 2.5 \times 2.5 \mathrm{~mm}^{2}$ are shown in Fig. 2. Figure 2(a) shows one typical cross sectional image (B scan) within the UHS-OMAG structural volume, which is identical to the conventional OCT image where typical morphological features, such as cranium and cortex, are visualized. Figure 2(b) gives the corresponding blood flow image obtained from UHSOMAG, where the microblood flows (e.g., pointed by arrows) originating from dura mater; the rich cortical blood flow signals are clearly identified as well. Because of its depthresolved nature, UHS-OMAG is able to separate blood flows in the meninges from those in the cortex. Considering that the dural plexus is a vascular network exclusively above the cortical plexus, the flow signals above the cortical layer were simply treated as the signals from the meningeal vessels, which were usually at depths from 100 to $200 \mu \mathrm{m}$ below the cranium surface. According to this feature, we applied the segmentation algorithm described in Ref. 11 to the 3-D OMAG flow data to separate the blood flow signals in the meninges from those in the cortex. The segmentation resulted in two volumetric flow images, one for the meninges and the other for the cortex. Then, the maximum amplitude projection was applied to each segmented meningeal and cortical volume, giving the blood flow distribution maps shown in Figs. 2(c) (meninges) and 2(d) (cortex), respectively. From Fig. 2(c), the meningeal blood vessels are seen to be tortuous. Further, the large vessels do not greatly diminish in size, in spite of giving off many side branches. More importantly, the UHS-OMAG is capable of visualizing the rich anastomotic as

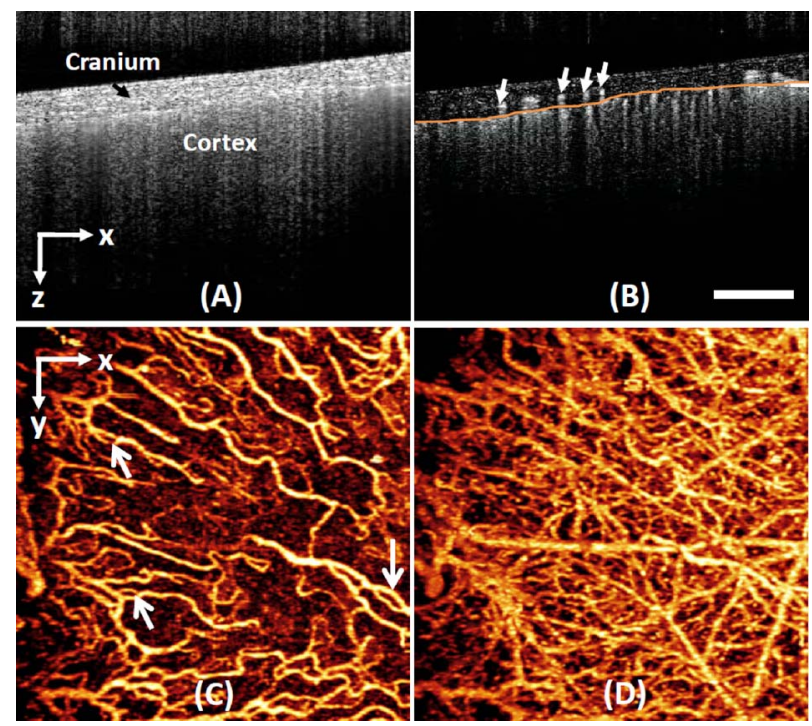

Fig. 2 Typical in vivo UHS-OMAG imaging of the microcirculation of the adult mouse brain with skull intact. (a) One typical UHS-OMAG cross sectional imaging (B scan) of microstructures showing morphological features, such as cranium and cortex, and (b) the corresponding depth-resolved blood flow image. The projection maps of blood flow network within (c) the meninges and (d) the cortex, respectively, are obtained from one 3-D scan. White bar $=500 \mu \mathrm{m}$. 


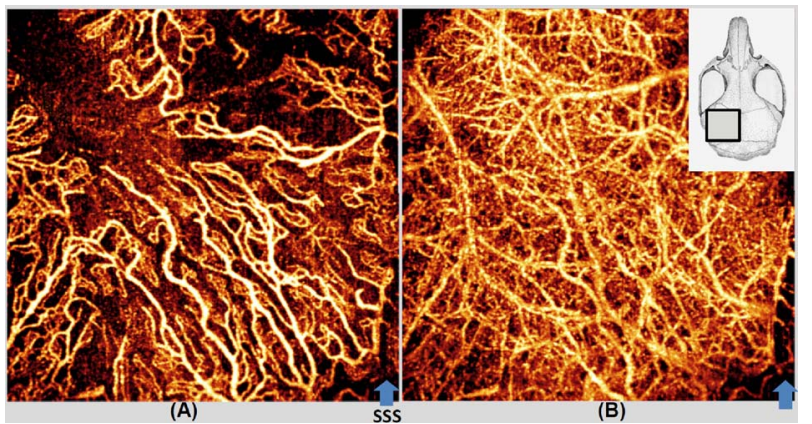

Fig. 3 Wide-field view $(4.5 \times 4.5 \mathrm{~mm})$ of the functional blood flow within (a) the meninges and (b) the cortex, respectively. The insert in the upper right corner is a sketch of the mouse skull where the marked box indicates the region imaged by UHS-OMAG. SSS is the superior sagittal sinus.

well as the splitting vessels (e.g., pointed by the arrows) in the meninges. These appearances of meningeal vessels directly result from the characteristic slow blood circulation in the intracranial dura matter. These findings from UHS-OMAG correspond well to standard dural histopathology, called primary or secondary anastomotic networks. ${ }^{13}$ Because of its capability of imaging much slower blood flows, it is not surprising that UHS-OMAG gave an image of greater vascular density for the cortex [Fig. 2(d)] compared to the conventional OMAG.

UHS-OMAG imaging through bone, in contrast to multiphoton microcopy ${ }^{14}$ and laser speckle imaging, ${ }^{3}$ is useful for obtaining functional information about tissue perfusion in the meninges. The results indicate that UHS-OMAG can be a useful tool to investigate the meningeal microcirculation involved with neurovascular disorders, in which changes in blood flow play an important role in the etiology, pathogenesis, prognosis, and responses to treatment. In some cases, it would be desirable to examine changes in meningeal blood flow globally as well as in individual vessels over the intracranial dural matter to elucidate how the brain functions to regulate meningeal blood flow in response to neurological diseases. To evaluate the feasibility of using UHS-OMAG as a useful tool in characterizing meningeal blood flow over a larger area of dural matter, we collected multiple 3-D images of the mouse brain over different regions on the left hemisphere. UHS-OMAG images from different regions were then combined as a mosaic (Fig. 3). UHS-OMAG imaging results were striking; they provided volumetric measurements of detailed microcirculation through the vascular tree down to the capillary level with the skull left intact, without the need for dye injections, contrast agents, or surgical craniotomy. The meningeal vessels are seen to distribute unevenly as they course over the dural surface, and cross-linked blood vessels are directed more toward the posterior than the anterior segment [Fig. 3(a)]. These features from meningeal vessels do not resemble those of cortical vessel distribution [Fig. 3(b)]. Such appearances observed from UHS-OMAG are consistent with the vascular convolution described in the well-known references, ${ }^{1,13}$ demonstrating the power of UHS-OMAG in in- vestigations of neurological diseases and complications that may involve meningeal microcirculations, for example, migraine.

In summary, we highlight the improved flow sensitivity made with UHS-OMAG for imaging detailed perfusion distributions within meninges and the cortex. By applying previous OMAG algorithms on the slow scanning axis, we show that the UHS-OMAG system is sensitive to capillary level microcirculation in the meninges in mice with the cranium left intact. More important, the functional meningeal vessels are imaged without contamination from microcirculations from the cortical vessels. Therefore, this in vivo microangiographic imaging method promises the further improved understanding of dura-related neurovascular diseases.

\section{Acknowledgments}

This work was supported in part by research grants from the National Heart, Lung, and Blood Institute (R01 HL093140), National Institute of Biomedical Imaging and Bioengineering (R01 EB009682), and the American Heart Association (0855733).

\section{References}

1. G. F. Rowbotham and E. Little, "Circulation of the cerebral hemispheres," Br. J. Surg. 52, 8-21 (1965).

2. G. F. Rowbotham and E. Little, "New concepts on the etiology and vascularization of meningioma; the mechanisms of migraine; the chemical processes of the cerebrospinal fluid; and the formation of collections of blood or fluid in the subdural space," Br. J. Surg. 52, 21-24 (1965).

3. H. Bolay, U. Reuter, A. K. Dunn, Z. Huang, D. A. Boas, and M. A Moskowitz, "Intrinsic brain activity triggers trigeminal meningeal afferents in a migraine model," Nat. Med. 8, 136-142 (2002).

4. B. Depreitere, C. Van Lierde, J. Vander Sloten, R. Van Audekercke, G. Van Der Perre, C. Plets, and J. Goffin, "Mechanics of acute subdural hematomas resulting from bridging vein rupture," J. Neurosurg. 104, 950-956 (2006)

5. R. K. Wang, S. L. Jacques, Z. Ma, S. Hurst, S. R. Hanson, and A. Gruber, "Three dimensional optical angiography," Opt. Express 15(7), 4083-4097 (2007)

6. A. F. Fercher, W. Drexler, C. K. Hitzenberger, and T. Lasser, "Optical coherence tomography-principles and applications," Rep. Prog. Phys. 66, 239-303 (2003).

7. P. H. Tomlins and R. K. Wang, "Theory, development and applications of optical coherence tomography," J. Appl. Phys., J. Phys. D 38, 2519-2535 (2005).

8. Y. L. Jia, N. J. Alkayed, and R. K. Wang, "The potential of optical micro-angiography to monitor cerebral blood perfusion and vascular plasticity following traumatic brain injury in mice in vivo," $J$. Biomed. Opt. 14, 040505 (2009).

9. R. K. Wang, "Directional blood flow imaging in volumetric optical micro-angiography achieved by digital frequency modulation," Opt. Lett. 33(16) 1878-1880 (2008).

10. R. K. Wang, "Optical microangiography: a label free 3D imaging technology to visualize and quantify blood circulations within tissue beds in vivo," IEEE J. Sel. Top. Quantum Electron. (in press).

11. R. K. Wang and L. An, "Doppler optical micro-angiography for volumetric imaging of vascular perfusion in vivo," Opt. Express 17, 8926-8940 (2009).

12. H. H. Lipowsky, "Microvascular rheology and hemodynamics," Microcirculation 12, 5-15 (2005).

13. C. W. Kerber and T. H. Newton, "The macro and microvasculature of the dura mater," Neuroradiology 6, 175-179 (1973).

14. T. Misgeld and M. Kerschensteiner, "In vivo imaging of the diseased nervous system," Nat. Rev. Neurosci. 7, 449-463 (2006). 